\title{
Alternative Splicing of Key Genes in LOX Pathway Involves Biosynthesis of Volatile Fatty Acid Derivatives in Tea Plant (Camellia sinensis)
}

Qingshan $\mathrm{Xu}^{1}$, Long Cheng ${ }^{1}$, Yu $\mathrm{Mei}^{2}$, Linli Huang ${ }^{1}$, Junyan $\mathrm{Zhu}^{2}$, Xiaozeng $\mathrm{Mi}^{2}$, Youben $\mathrm{Yu}^{1 *}$, Chaoling $\mathrm{Wei}^{2 *}$

${ }^{1}$ College of Horticulture, Northwest A\&F University, Yangling 712100, Shaanxi, China

${ }^{2}$ State Key Laboratory of Tea Plant Biology and Utilization, Anhui Agricultural University, Hefei, Anhui, China

*Corresponding Author: weichl@ahau.edu.cn; chyyjs@nwsuaf.edu.cn. 


\section{Supporting information}

Table S1 Meteorological parameters of the 916 Tea Plantation in 2015.

\begin{tabular}{cccc}
\hline Time point & Average temperature $\left({ }^{\circ} \mathbf{C}\right)$ & Precipitation $(\mathbf{m m})$ & Duration of sunshine (h) \\
\hline April & 16.4 & 115.4 & 181.9 \\
June & 28.26 & 176.3 & 245.2 \\
August & 26.9 & 147.8 & 176.6 \\
September & 22.9 & 41.6 & 163.4 \\
October & 17.6 & 56.7 & 158.7 \\
\hline
\end{tabular}

Table S2 Standard curves for the major volatiles established using a series of diluted solutions of authentic compounds.

\begin{tabular}{ccr}
\hline Compounds & Formula & $\mathbf{R}^{2}$ \\
\hline cis-3-hexen-1-ol & $\mathrm{Y}=4 \mathrm{E}-6 \mathrm{X}+1.8449$ & 0.9534 \\
cis-3-hexenyl esters & $\mathrm{Y}=1 \mathrm{E}-6 \mathrm{X}+0.8646$ & 0.9865 \\
trans-2-hexenyl esters & $\mathrm{Y}=1 \mathrm{E}-6 \mathrm{X}-0.9909$ & 0.9979 \\
\hline
\end{tabular}

Note: $\mathrm{Y}$ is the amount $\left(\mu \mathrm{g} \mathrm{kg}^{-1}\right)$ of VFADs based on the peak area $\mathrm{X}$ generated using GC-MS.

Table S3 Plant lipoxygenase sequences used for $C s L O X$ gene Prediction

\begin{tabular}{|c|c|c|}
\hline Organism & Sequence ID & GeneBank Accession \\
\hline & $A t L O X 1$ & Q06327 \\
\hline & AtLOX2 & P38418 \\
\hline \multirow[t]{4}{*}{ Arabidopsis thaliana } & AtLOX3 & Q9SMW1 \\
\hline & AtLOX4 & Q9FNX8 \\
\hline & AtLOX5 & Q9FNX7 \\
\hline & AtLOX6 & Q9CAG3 \\
\hline \multirow[t]{10}{*}{ Actinidia deliciosa } & $A d L O X 2$ & DQ497797 \\
\hline & PtLOX1 & XP_002299250.1 \\
\hline & PtLOX2 & XP_002297796.2 \\
\hline & PtLOX3 & XP_006368564.1 \\
\hline & PtLOX4 & XP_006388115.1 \\
\hline & PtLOX5 & XP_006369132.1 \\
\hline & PtLOX6 & XP_002304125.1 \\
\hline & PtLOX7 & XP_006382593.1 \\
\hline & PtLOX8 & XP_006382594.1 \\
\hline & PtLOX9 & XP_011036799.1 \\
\hline \multirow[t]{8}{*}{ Populus trichocarpa } & PtLOX10 & XP_006382595.1 \\
\hline & PtLOX11 & XP_002311617.1 \\
\hline & PtLOX12 & XP_002311724.1 \\
\hline & PtLOX13 & XP_002314229.2 \\
\hline & PtLOX14 & XP_002314548.2 \\
\hline & PtLOX15 & XP_002315780.1 \\
\hline & PtLOX16 & XP_002319014.2 \\
\hline & PtLOX17 & XP_002319015.2 \\
\hline
\end{tabular}




\begin{tabular}{|c|c|c|}
\hline & PtLOX18 & XP_002320037.2 \\
\hline & PtLOX19 & XP_002320571.2 \\
\hline & PtLOX20 & XP_002323952.2 \\
\hline & $V v L O X A$ & XP_002285574 \\
\hline & $V v L O X C$ & FJ858257 \\
\hline & $V v L O X G$ & XP_002283147 \\
\hline & $V \nu L O X H$ & XP_002283135 \\
\hline & $V v L O X I$ & XP_002283123 \\
\hline \multirow[t]{9}{*}{ Vitis vinifera } & $V v L O X J$ & XP_002263854 \\
\hline & $V v L O X L$ & XP_002278007 \\
\hline & $V v L O X P$ & XP_002265505 \\
\hline & $V v L O X T$ & XP_010651289.1 \\
\hline & $V v L O X U$ & XP_010659819.1 \\
\hline & $V v L O X V$ & XP_010659859.1 \\
\hline & $V \nu L O X W$ & NP_001290017.1 \\
\hline & $C s L O X 2$ & FJ418174.1 \\
\hline & CsLOX3 & FJ794853.1 \\
\hline \multirow[t]{5}{*}{ Camellia sinensis } & CsLOX4 & MG708225 \\
\hline & CsLOX5 & MG708226 \\
\hline & CsLOX6 & MG708227 \\
\hline & CsLOX7 & MG708228 \\
\hline & CsLOX11 & MG708232 \\
\hline
\end{tabular}

Table S4 Gene-specific primers of splicing events

\begin{tabular}{lll}
\hline \multicolumn{1}{c}{ Name } & Sequence (5'-3') & Comment \\
\hline CsLOX3-iso1-F & TCAAACTTGCTTTACCTTCC & cDNA amplification \\
CsLOX3-iso1-R & CCTGAAAATGTTAGCTGC & cDNA amplification \\
CsLOX3-iso2-F & CCATTGATTCCTTGTTCG & cDNA amplification \\
CsLOX3-iso2-R & CCTACCGTTCGGATTTC & cDNA amplification \\
CsLOX3-iso3-F & TCAAACTTGCTTTACCTTCC & cDNA amplification \\
CsLOX3-iso3-R & CTTCATGGCCATTCCTTA & cDNA amplification \\
CsLOX6-iso2-F & ATGGTTTAGGGACGAGGG & cDNA amplification \\
CsLOX6-iso2-R & ATCAACAGTTCTACCTCC & cDNA amplification \\
CsLOX6-iso3-F & GCACTCGTAGAAAGAATGG & cDNA amplification \\
CsLOX6-iso3-R & GTCGGTATTTTCCTCCTG & cDNA amplification \\
CsADH8-iso1-F & AGGTGAGGTGGTTGAGC & cDNA amplification \\
CsADH8-iso1-R & AACAGCACGCCAACAATAC & cDNA amplification \\
CsADH8-iso2-F & ATCAAAGATTTCCAGTCGG & cDNA amplification \\
CsADH8-iso2-R & CAGCAGAGTGCGTAAGC & cDNA amplification \\
CsADH4-iso3-F & TTTGTTGGTATGGCGGTG & cDNA amplification \\
CsADH4-iso3-R & CTTACAACAAGTGGTCAGG & cDNA amplification \\
AsODN-CsLOX4-iso1 & GGAAGAAGGTTAACTCCTGA & AsODN experiment \\
ODN-CsLOX4-iso1 & TCAGGAGTTAACCTTCTTCC & AsODN experiment \\
AsODN-CsLOX4-iso2 & CTGAGTATACGGGCATGCCT & AsODN experiment \\
ODN-CsLOX4-iso2 & AGGCATGCCCGTATACTCAG & AsODN experiment \\
AsODN-CsADH4-iso3 & TGCTTAGGTGAGCACAGCTC & AsODN experiment
\end{tabular}




\begin{tabular}{lll}
\hline ODN-CsADH4-iso3 & GAGCTGTGCTCACCTAAGCA & AsODN experiment \\
CsLOX4-iso1-qF & GTGAACAAAGTAAGAGAGAGC & qPCR \\
CsLOX4-iso1-qR & TTATGTAAGGCTCTGTCACG & qPCR \\
CsLOX4-iso2-qF & CACTGCCATTGATTCCTTG & qPCR \\
CsLOX4-iso2-qR & TGGTGATTGCCGATTCTG & qPCR \\
CsADH4-iso3-qF & TCCTGGTTCTCCTATAACTG & qPCR \\
CsADH4-iso3-qR & CAACATAGGCCGTCATAC & qPCR \\
\hline
\end{tabular}

Table S5 Correlation analysis of climatic parameters, transcript expression levels and VFAD contents

\begin{tabular}{|c|c|c|c|c|c|}
\hline Gene name & (Z)-3-hexenol & (Z)-3-hexenyl esters & $(E)$-2-hexenyl esters & JA & MeJA \\
\hline Temperature & -0.673 & -0.212 & 0.800 & 0.620 & 0.232 \\
\hline Precipitation & -0.966 & -0.809 & 0.322 & 0.239 & -0.630 \\
\hline Light & -0.927 & -0.861 & 0.062 & 0.504 & -0.441 \\
\hline CsLOX2-iso1 & -0.300 & -0.215 & 0.012 & 0.873 & 0.384 \\
\hline CsLOX2-iso2 & -0.290 & -0.292 & -0.126 & 0.927 & 0.266 \\
\hline CsLOX2-iso3 & 0.137 & 0.095 & -0.025 & 0.376 & 0.066 \\
\hline CsLOX3 & -0.299 & -0.564 & -0.586 & 0.637 & -0.170 \\
\hline CsLOX4-iso1 & 0.726 & 0.424 & -0.611 & -0.423 & 0.261 \\
\hline CsLOX4-iso2 & 0.749 & 0.348 & -0.769 & -0.426 & 0.121 \\
\hline CsLOX4-iso3 & -0.271 & -0.389 & -0.315 & 0.863 & 0.080 \\
\hline CsLOX5 & -0.290 & -0.413 & -0.131 & -0.737 & -0.787 \\
\hline CsLOX7 & 0.453 & 0.369 & -0.227 & 0.585 & 0.601 \\
\hline CsAOC1 & 0.838 & 0.692 & -0.211 & -0.844 & 0.180 \\
\hline CsAOC2 & 0.441 & 0.111 & -0.560 & -0.897 & -0.351 \\
\hline CsOPR1 & 0.632 & 0.150 & -0.906 & -0.243 & -0.044 \\
\hline CsOPR2 & 0.414 & 0.252 & -0.393 & 0.571 & 0.520 \\
\hline CsOPR3 & -0.117 & 0.304 & 0.865 & -0.164 & 0.148 \\
\hline CsOPR4 & 0.114 & -0.202 & -0.446 & -0.618 & -0.709 \\
\hline CsJMT & 0.517 & 0.420 & -0.162 & 0.301 & 0.374 \\
\hline CsHPL & 0.746 & 0.983 & 0.432 & -0.204 & 0.846 \\
\hline CsADH1 & 0.301 & -0.082 & -0.800 & 0.286 & 0.093 \\
\hline $\mathrm{CsADH} 2$ & -0.078 & 0.285 & 0.657 & 0.677 & 0.560 \\
\hline CsADH3 & 0.526 & 0.627 & 0.068 & 0.130 & 0.832 \\
\hline CsADH4-iso1 & 0.644 & 0.629 & -0.129 & -0.097 & 0.703 \\
\hline CsADH4-iso2 & 0.375 & 0.219 & -0.342 & 0.573 & 0.410 \\
\hline CsADH4-iso3 & 0.887 & 0.846 & -0.133 & -0.209 & 0.759 \\
\hline CsADH5 & 0.610 & 0.766 & 0.206 & -0.121 & 0.821 \\
\hline CsADH6 & 0.007 & 0.442 & 0.877 & -0.227 & 0.276 \\
\hline CsADH7 & 0.260 & -0.270 & -0.984 & -0.339 & -0.459 \\
\hline CsADH8-iso1 & 0.087 & -0.220 & -0.682 & 0.127 & 0.001 \\
\hline CsADH8-iso2 & -0.760 & -0.506 & 0.551 & -0.039 & -0.529 \\
\hline CsADH10-iso1 & 0.634 & 0.455 & -0.436 & -0.050 & 0.534 \\
\hline CsADH10-iso2 & 0.439 & 0.232 & -0.329 & 0.142 & 0.071 \\
\hline
\end{tabular}




\begin{tabular}{llllll}
\hline CsADH10-iso3 & 0.683 & 0.946 & 0.447 & -0.007 & 0.942 \\
CsADH10-iso4 & -0.182 & -0.184 & -0.160 & 0.567 & 0.321 \\
CsADH11 & -0.221 & -0.686 & -0.815 & -0.220 & -0.856 \\
CsADH12 & 0.528 & 0.059 & -0.913 & -0.081 & -0.007 \\
CsADH13 & 0.538 & 0.372 & -0.396 & -0.297 & 0.362 \\
\hline
\end{tabular}

Note: the value in red or green represent a positive and negative correlations, respectively.

Table S6 Genes related to VFAD biosynthesis.

\begin{tabular}{cl}
\hline Name & Gene id \\
\hline CsLOX2 & TEA009423.1 \\
CsLOX3 & FJ794853.1 \\
CsLOX4 & TEA011776.1 \\
CsLOX5 & TEA020304.1 \\
CsLOX6 & TEA020832.1 \\
CsLOX7 & TEA012289.1 \\
CsLOX11 & TEA027370.1 \\
CsAOC1 & TEA012644.1 \\
CsAOC2 & TEA020075.1 \\
CsOPR1 & TEA025907.1 \\
CsOPR2 & TEA029800.1 \\
CsOPR3 & TEA027519.1 \\
CsOPR4 & TEA026804.1 \\
CsJMT & TEA031418.1 \\
CsHPL & TEA008699.1 \\
CsADH1 & TEA024401.1 \\
CsADH2 & TEA024406.1 \\
CsADH3 & TEA032488.1 \\
CsADH4 & TEA033182.1 \\
CsADH5 & TEA022425.1 \\
CsADH6 & TEA029320.1 \\
CsADH7 & TEA029314.1 \\
CsADH8 & TEA018669.1 \\
CsADH9 & TEA016726.1 \\
CsADH10 & TEA015686.1 \\
CsADH11 & TEA033678.1 \\
CsADH12 & TEA010675.1 \\
CsADH13 & TEA029515.1 \\
CsAAT & TEA011609.1 \\
\hline & \\
\hline
\end{tabular}


replicate 1

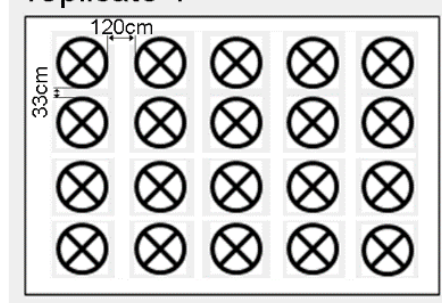

replicate 2

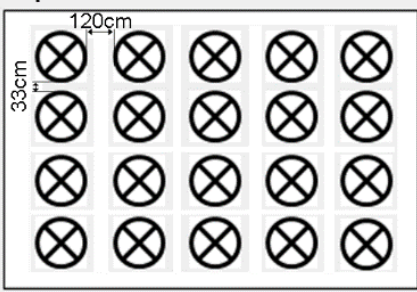

replicate 3

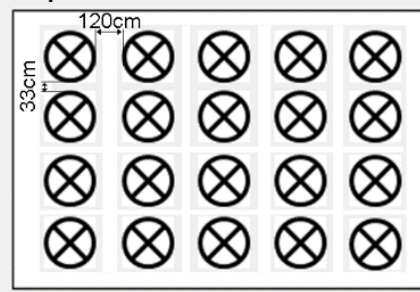

Figure S1. A schematic view of the experimental design $\otimes$ : tea plant. 20 plants were planted in an experimental plot with a $120 \mathrm{~cm}$ row distance and $33 \mathrm{~cm}$ space between plants within a row, and three experimental plots, including 60 plants, were carried out in this study.

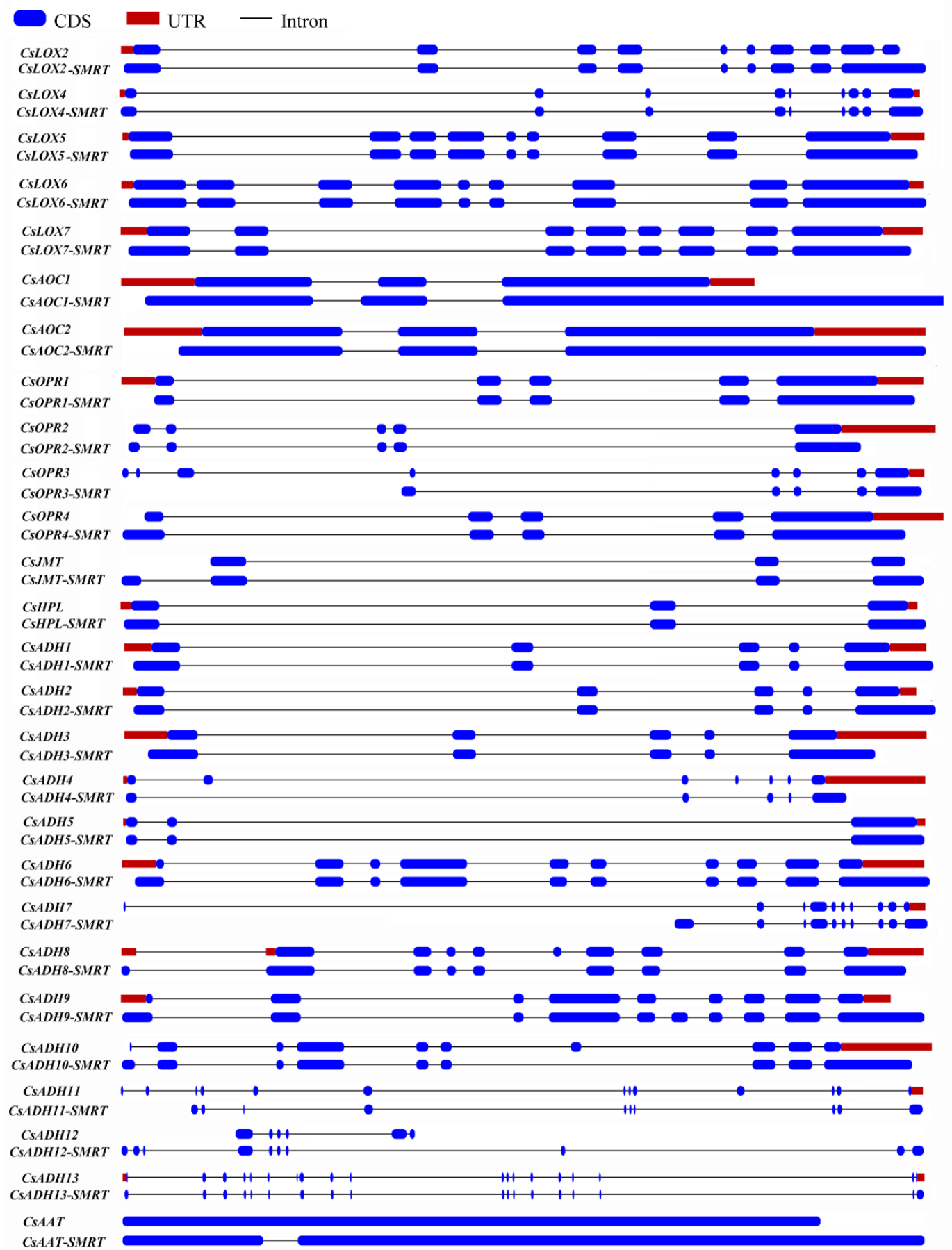


Figure S2. The 18 identified full-length transcripts from SMRT sequencing data align with the gene from genome annotation.

\section{CSLOX2}

CsLOX2-iso2 TTTTTGACAGATCTACTTCCCAGACTTGTCAAGTTCATTAGTGATACTGAAGAAGCCATC CsLOX2-iso3 TTTTTGACAGATCTACTTCCCAGACTTGTCAAGTTCATTAGTGATACTGAAGAAGCCATC CsL0X2-iso2/M13

CsLOX2-iso3/M13

CsLOX2-iso2

CsLOX2-i so3

TTGCGCTTTGAGACCCCTGCACTCGTAGAAAAAGACAAATTTTCATGGTTTAGGGACGAG CsLOX2-iso2/M13

CsLOX2-i so3/M13

TTGCGCTTTGAGACCCCTGCACTCGTAGAAA-

CsLOX2-iso2

CsLOX2-iso3

CsL0X2-iso2/M13 GAATTCTCCCGACAAACGTTGGCTGGTCTAAACCCATATAGCATACAATTGGTCAACGAA

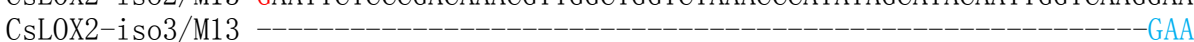

CsLOX2-iso2 TGGCCATTGAGGAGTAAACTCGACCCTAAGATCTATGGGGCTCCCGAATCTGCTATTACC CsLOX2-i so3 TGGCCATTGAGGAGTAAACTCGACCCTAAGATCTATGGGGCTCCCGAATCTGCTATTACC CsLOX2-i so2/M13 TGGCCATTGAGGAGTAAACTCGACCCTAAGATCTATGGGGCTCCCGAATCTGCTATTACC CsL0X2-i so3/M13 TGGCCATTGAGGAGTAAACTCGACCCTAAGATCTATGGGGCTCCCGAATCTGCTATTACC

CsLOX2-iso2 AAAGATCTTATTGAGCGAGAGATCAAAGGCATGATCACCCTTGAAGAGGCATTGCAACAA CsLOX2-i so3 AAAGATCTTATTGAGCGAGAGATCAAAGGCATGATCACCCTTGAAGAGGCATTGCAACAA CsLOX2-i so2/M13 AAAGATCTTATTGAGCGAGAGATCAAAGGCATGATCACCCTTGAAGAGGCATTGCAACAA CsLOX2-iso3/M13 AAAGATCTTATTGAGCGAGAGATCAAAGGCATGATCACCCTTGAAGAGGCATTGCAACAA

CsLOX2-iso2 AAGAAGCTATTCATGCTGGATTACCATGACTTGCTCTTACCATATGTGAAGAAAGTGAGA CsLOX2-iso3 AAGAAGCTATTCATGCTGGATTACCATGACTTGCTCTTACCATATGTGAAGAAAGTGAGA CsLOX2-i so2/M13 AAGAAGCTATTCATGCTGGATTACCATGACTTGCTCTTACCATATGTGAAGAAAGTGAGA CsL0X2-i so3/M13 AAGAAGCTATTCATGCTGGATTACCATGACTTGCTCTTACCATATGTGAAGAAAGTGAGA

CsLOX2-i so2 GAGATAGAAGGGACTACACTCTATGGATCCAGGACCTTGTTCTTCCTGACACCAAATGGC CsLOX2-i so3 GAGATAGAAGGGACTACACTCTATGGATCCAGGACCTTGTTCTTCCTGACACCAAATGGC CsLOX2-i so2/M13 GAGATAGAAGGGACTACACTCTATGGATCCAGGACCTTGTTCTTCCTGACACCAAATGGC CsL0X2-i so3/M13 GAGATAGAAGGGACTACACTCTATGGATCCAGGACCTTGTTCTTCCTGACACCAAATGGC

CsLOX2-i so2 ACGTTGAGGCCGTTGGCGATTGAGTTAACTCGGCCGCCCATGGACGGTAAGGCTGAGTGG CsLOX2-i so3 ACGTTGAGGCCGTTGGCGATTGAGTTAACTCGGCCGCC-ATGGACGGTAAGGCTGAGTGG CsL0X2-i so2/M13 ACGTTGAGGCCGTTGGCGATTGAGTTAACTCGGCCGCCCATGGACGGTAAGGCTGAGTGG CsLOX2-i so3/M13 ACGTTGAGGCCGTTGGCGATTGAGTTAACTCGGCCGCCCATGGACGGTAAGGCTGAGTGG

CsLOX2-i so2 AAGCAAGTGTTCACTCCAACATGGGATGCTACAGGTTGCTGGCTATGGAGGCTTGCTAAG CsLOX2-i so3 AAGCAAGTGTTCACTCCAACATGGGATGCTACAGGTTGCTGGCTATGGAGGCTTGCTAAG CsLOX2-i so2/M13 AAGCAAGTGTTCACTCCAACATGGGATGCTACAGGTTGCTGGCTATGGAGGCTTGCTAAG CsL0X2-i so3/M13 AAGCAAGTGTTCACTCCAACATGGGATGCTACAGGTTGCTGGCTATGGAGGCTTGCTAAG

CsLOX2-iso2 ACTCATGTTCTTGCTCATGACTCTGGCTATCACCAGCTTGTTAGTCACTGGTTAAGAACA CsLOX2-iso3 ACTCATGTTCTTGCTCATGACTCTGGCTATCACCAGCTTGTTAGTCACTGGTTAAGAACA CsL0X2-i so2/M13 ACTCATGTTCTTGCTCATGACTCTGGCTATCACCAGCTTGTTAGTCACTGGTTAAGAACA CsLOX2-i so3/M13 ACTCATGTTCTTACTCATGACTCTGGCTATCACCAGCTTGTTAGTCACTGGTTAAGAACA

CsLOX2-iso2 CACTGTGCGACAGAGCCATACATAATAGCGAGCAACAGGCAACTGAGCGCGATGCACCCC CsLOX2-i so3 CACTGTGCGACAGAGCCATACATAATAGCGAGCAACAGGCAACTGAGCGCGATGCACCCC CsLOX2-i so2/M13 CACTGTGCGACAGAGCCATACATAATAGCGAGCAACAGGCAACTGAGCGCGATGCACCCC CsL0X2-i so3/M13 CACTGTGCGACAGAGCCATACATAATAGCGAGCAACAGGCAACTGAGCGCGATGCACCCC CsLOX2-i so2 ATTTACAGACTACTGCACCCTCATTTCCGGTACACGATGGAGATCAATGCTCTCGCTCGT CsLOX2-iso3 ATTTACAGACTACTGCACCCTCATTTCCGGTACACGATGGAGATCAATGCTCTCGCTCGT CsLOX2-i so2/M13 ATTTACAGACTACTGCACCCTCATTTCCGGTACACGATGGAGATCAATGCTCTCGCTCGT CsL0X2-iso3/M13 ATTTACAGACTACTGCACCCTCATTTCCGGTACACGATGGAGATCAATGCTCTCGCTCGT

CsLOX2-iso2 GAAGCTCTCATCAATGCCAAAGGGATCATCGAGACATCTTTCTCCCCTGCCAAGTATAGC CsLOX2-i so3 GAAGCTCTCATCAATGCCAAAGGGATCATCGAGACATCTTTCTCCCCTGCCAAGTATAGC CsLOX2-i so2/M13 GAAGCTCTCATCAATGCCAAAGGGATCATCGAGACATCTTTCTCCCCTGCCAAGTATAGC CsLOX2-i so3/M13 GAAGCTCTCATCAATGCCAAAGGGATCATCGAGACATCTTTCTCCCCTGCCAAGTATAGC 
CsLOX2-iso2 ATGGAGCTTAGCTCTGTTGCCTACGACCAACAATGGCGGTTCGACCACCAGGCTCTTCCA CsLOX2-i so3 ATGGAGCTTAGCTCTGTTGCCTACGACCAACAATGGCGGTTCGACCACCAGGCTCTTCCA CsLOX2-iso2/M13 ATGGAGCTTAGCTCTGTTGCCTACGACCAACAATGGCGGTTCGACCACCAGGCTCTTCCA CsLOX2-iso3/M13 ATGGAGCTTAGCTCTGTTGCCTACGACCAACAATGGCGGTTCGACCACCAGGCTCTTCCA

CsLOX2-iso2 GCTGACTTAATTAGCAGGGGAATGGCTGTGGAAGATCCAACCTCTCCTCATGGCCTAAAG CsLOX2-i so3 GCTGACTTAATTAGCAGGGGAATGGCTGTGGAAGATCCAACCTCTCCTCATGGCCTAAAG CsLOX2-i so $2 /$ M13 GCTGACTTAATTAGCAGGGGAATGGCTGTGGAAGATCCAACCTCTCCTCATGGCCTAAAG CsLOX2-i so3/M13 GCTGACTTAATTAGCAGGGGAATGGCTGTGGAAGATCCAACCTCTCCTCATGGCCTAAAG

CsLOX2-i so2 CTAACAATAGAGGACTACCCTTTTGCCAATGATGGTCTTGTTCTCTGGGATGCCATCAAA CsLOX2-i so 3 CTAACAATAGAGGACTACCCTTTTGCCA-TGATGGTCTTGTTCTCTGGGATGCCATCAAA CsLOX2-i so2/M13 CTAACAATAGAGGACTACCCTTTTGCCAATGATGGTCTTGTTCTCTGGGATGCCATCAAA CsL0X2-iso3/M13 CTAACAATAGAGGACTACCCTTTTGCCAATGATGGTCTTGTTCTCTGGGACGCCATCAAA

CsLOX2-iso2 CAGTGGGTTACAGACTATGTCAAACACTATTACCCAGATCCAAGCTTCATAAAGTCCGAC CsLOX2-iso3 CAGTGGGTTACAGACTATGTCAAACACTATTACCCAGATCCAAGCTTCATAAAGTCCGAC CsLOX2-i so2/M13 CAGTGGGTTACAGACTATGTCAAACACTATTACCCAGATCCAAGCTTCATAAAGTCCGAC CsLOX2-i so3/M13 CAGTGGGTTACAGACTATGTCAAACACTATTACCCAGATCCAAGCTTCATAAAGTCCGAC

CsLOX2-iso2 GAAGAGCTTCAATCATGGTGGACAGAGATCCGAACAGTCGGTCACCAAGACAAAAAGGAC CsLOX2-i so3 GAAGAGCTTCAATCATGGTGGACAGAGATCCGAACAGTCGGTCACCAAGACAAAAAGGAC CsL0X2-i so2/M13 GAAGAGCTTCAATCATGGTGGACAGAGATCCGAACAGTCGGTCACCAAGACAAAAAGGAC CsL0X2-i so3/M13 GAAGAGCTTCAATCATGGTGGACAGAGATCCGAACAGTCGGTCACCAAGACAAAAAGGAC

CsLOX2-i so2 GATCCGTGGTGGCCGGTTCTGAAAACCCCTGAGGACCTCATCGGCATTCTCACAACCATG CsLOX2-i so3 GATCCGTGGTGGCCGGTTCTGAAAACCCCTGAGGACCTCATCGGCATTCTCACAACCATG CsLOX2-i so2/M13 GATCCGTGGTGGCCGGTTTTGAAAACCCCTGAGGACCTCATCGGCATTCTCACAACCATG CsLOX2-iso3/M13 GATCCGTGGTGGCCGGTTTTGAAAACCCCTGAGGACCTCATCGGCATTCTCACAACCATG

CsLOX2-iso2 ATCTGGGTAGCCTCCGGTCACCACTCTGCAGTCAACTTTGGCCAATATGCTTTCGCAGGT CsLOX2-i so3 ATCTGGGTAGCCTCCGGTCACCACTCTGCAGTCAACTTTGGCCAATATGCTTTCGCAGGT CsLOX2-i so2/M13 ATCTGGGTAGCCTCCGGTCACCACTCTGCAGTCAACTTTGGCCAATATGCTTTCGCAGGT CsLOX2-i so3/M13 ATCTGGGTAGCCTCCGGTCACCACTCTGCAGTCAACTTTGGCCAATATGCTTTCGCAGGT

CsLOX2-i so2 TATTTCCCGAACAGGCCAACCATTGCCAGGAGGAAAATACCGACAGAAGATCCATCGGAG CsLOX2-i so3 TATTTCCCGAACAGGCCAACCATTGCCAGGAGGAAAATACCGACAGAAGATCCATCGGAG CsLOX2-i so2/M13 TATTTCCCGAACAGGCCAACCATTGCCAGGAGGAAAATACCGACAGAAGATCCATCGGAG CsL0X2-i so3/M13 TATTTCCCGAACAGGCCAGCCATTGCCAGGAGGAAAATACCGAC

CsLOX2-iso2 CAAGAGTTGAAGAATTTCCTGAACAAGCCGGAGGTAGAACTGTTGATGAGCTTTCCTTCG CsLOX2-i so 3 CAAGAGTTGAAGAATTTCCTGAACAAGCCGGAGGTAGAACTGTTGATGAGCTTTCCTTCG CsL0X2-i so2/M13 CAAGAGTTGAAGAATTTCCTGAACAAGCCGGAGGTAGAACTGTTGAT-

CsLOX2-iso2 CA

CsLOX2-iso 3 CA

CsL0X2-iso2/M13 --

\section{CsLOX4}

CsLOX4-iso1

CsLOX4-iso2

-ACATCTATCCTACTGC-AAACACATCCTTTTTATAAGAGCCACTCAAA-CTTGCTTTAC

CsLOX4-iso3 ACCATCTATCCTACTGCTAAACACATCCTTTTTATAAGAGCCACTCAAAACTTGCTTTAC CsL0X4-iso1/M13

CsLOX4-i so3/M13 -ACATCTATCCTACTGC-AAACACATCCTTTTTATAAGAGCCACTCAAA-CTTGCTTTAC

CsLOX4-iso 1

CsLOX4-iso2

CsLOX4-iso3

CCACACCACCAAATTTAACTTATTCCAAACCCAGATCACGAAAAAAATAATCTCCAA CTTCCACACCACCAAATTTAACTTATTCCAAACCCAGATCACGAAAAAAATAATCTCCAG CTTCCACACCACCAAATTTAACTTATTCCAAACCCAGATCACGAAAAAAATAATTTCCACsLOX4-i so 1/M13 CTTTCACACCACCAAATTTAACTTATTCCAAACCCAGATCACGAAAAAAATAATCTCCACsLOX4-i so3/M13 CTTCCACACCACCAAATTTAACTTATTCCAAACCCAGATCACGAAAAAAATAATCTCCA-

CsLOX4-iso 1 AAAAAAAAAAAAAAAAAATGTTGAACAGTCAAGTTCACCAGTCTCATACTGTCCAAATT CsLOX4-i so2 AAAAAAAAAAAAAAAAAAATGTTGAACAGTCAAGTTCACCAGTCTCATACTGTCCAAATT CsLOX4-i so3 ---AAAAAAAAAAAAAAAATGTTGAACAGTCAAGTTCACCAGTCTCATACTGTCCAAATT CsLOX4-i so1/M13 ----AAAAAAAAAAAAAAATGTTGAACAGTCAAGTTCACCAGTCTCATACTGTCCAAATT CsLOX4-i so3/M13 ---AAAAAAAAAAAAAAAATGTTGAACAGTCAAGTTCACCAGTCTCATACTGTCCAAATT CTAATTCCATGGCACAAGCCATTCCCCTCCGGAACCGCTAGCCCTTCGTCGTCACTACAC 
CsLOX4-iso3 CTAATTCCATGGCACAAGCCATTCCCCTCCGGAACCGCTAGCCCTTCGTCGTCACTACAC CsLOX4-i so 1/M13 CTAATTCCATGGCACAAGCCATTCCCCTCCGGAACCGCTAGCCCTTCGCCGTCACTACAC CsL0X4-i so3/M13 CTAATTCCATGACACAAGCCATTCCCCTCCGGAACCGCTAGCCCTTCGTCGTCACTACAC

CsL0X4-iso 1 CsLOX4-iso2 CsLOX4-iso3 CTGCTGAAACCAGGGTTGTGTGGAAACCAAAAGGATAAAGGTCGTGTTCGGTGTGTTCCT CTGCTGAAACCAGGGTTGTGTGGAAACCAAAAGGATAAAGGTCGTGTTCGGTGTGTTCCT CTGCTGAAACCAGGGTTGTGTGGAAACCAAAAGGATAAAGGTCGTGTTCGGTGTGTTCCT CsLOX4-i so1/M13 CTGCTGAAACCAGGGTTGTGTGGAAACCAAAAGGATAAAGGTCGTGTTCGGTGTGTTCCT CsLOX4-i so3/M13 CTGCTGAAACCAGGGTTGTGTGGAAACCAAAAGGATAAAGGTCGTGTTCGGTGTGTTCCT

CsLOX4-iso1 CsLOX4-iso2 CsLOX4-iso3 AGCACCATTAAAGCCATAGCAACCACTACTACCGAGCAGACCACGATTGTTAAGGCTGTT AGCACCATTAAAGCCATAGCAACCACTACTACCGAGCAGACCACGATTGTTAAGGCTGTT AGCACCATTAAAGCCATAGCAACCACTACTACCGAGCAGACCACGATTGTTAAGGCTGTT CsLOX4-i so 1/M13 AGCACCATTAAAGCCATAGCAACCACTACTACCGAGCAGACCACGATTGTTAAGGCTGTT CsLOX4-i so3/M13 AGCACCATTAAAGCCATAGCAACCACTACTACCGAGCAGACCACGATTGTTAAGGCTGTT

CsLOX4-iso 1 CsLOX4-iso2 GTTTGTGTGAAATTAACAGTCGGTGGTTTCTTGTCGAACCTTGGATTAAGCCGTGGGCTT GTTTGTGTGAAATTAACAGTCGGTGGTTTCTTGTCGAACCTTGGATTAAGCCGTGGGCTT CsLOX4-i so3 GTTTGTGTGAAATTAACAGTCGGTGGTTTCTTGTCGAACCTTGGATTAAGCCGTGGGCTT CsLOX4-i so1/M13 GTTTGTGTGAAATTAACAGTCGGTGGTTTCTTGTCGAACCTTGGATTAAGCCGTGGGCTT CsLOX4-i so3/M13 GTTTGTGTGAAATTAACAGTCGGTGGTTTCTTGTCGAACCTTGGATTAAGCCGTGGGCTT

CsLOX4-iso 1 CsLOX4-i so2 GACGACGTTGCTGATATGCTTGGTAAATCAATCCAGTTGGAGCTTGTTAGTGCCGAGCTT GACGACGTTGCTGATATGCTTGGTAAATCAATCCAGTTGGAGCTTGTTAGTGCCGAGCTT CsLOX4-i so 3 GACGACGTTGCTGATATGCTTGGTAAATCAATCCAGTTGGAGCTTGTTAGTGCCGAGCTT CsLOX4-i so 1/M13 GACGACGTTGCTGATATGCTTGGTAAATCAATCCAGTTGGAGCTTGTTAGTGCCGAGCTT CsLOX4-i so3/M13 GACGACGTTGCTGATATGCTTGGTAAATCAATCCAGTTGGAGCTTGTTAGTGCCGAGCTT

CsLOX4-iso1 GATCCCAAGACGGGATTAGAGAAGGAAACAATAAAGGGGTATGCACATCGGACGAGCCAA CsLOX4-i so2 GATCCCAAGACGGGATTAGAGAAGGAAACAATAAAGGGGTATGCACATCGGACGAGCCAA CsLOX4-i so3 GATCCCAAGACGGGATTAGAGAAGGAAACAATAAAGGGGTATGCACATCGGACGAGCCAA CsL0X4-i so1/M13 GATCCCAAGACGGGATTAGAGAAGGAAACAATAAAGGGGTATGCACATCGGACGAGCCAA CsLOX4-i so3/M13 GATCCCAAGACGGGATTAGAGAAGGAAACAATAAAGGGGTATGCACATCGGACGAGCCAA

CsLOX4-iso 1 CsLOX4-iso2 CsLOX4-iso3 GAGGAGGATGAAGTGAAGTATGAATGCAATTTTGTAATTCCAGAAGGTTATGGTGAGATT GAGGAGGATGAAGTGAAGTATGAATGCAATTTTGTAATTCCAGAAGGTTATGGTGAGATT GAGGAGGATGAAGTGAAGTATGAATGCAATTTTGTAATTCCAGAAGGTTATGGTGAGATT CsLOX4-i so1/M13 GAGGAGGATGAAGTGAAGTATGAATGCAATTTTGTAATTCCAGAAGGTTATGGTGAGATT CsLOX4-iso3/M13 GAGGAGGATGAAGTGAAGTATGAATGCAATTTTGTAATTCCAGAAGGTTATGGTGAGATT

CsLOX4-iso 1 CsLOX4-iso2 CsLOX4-iso3 GGTGCAGTCTTGGTCGAGAATGAACACCACAAGGAAATGTACCTCAAGCAAATTGTCTTT GGTGCAGTCTTGGTCGAGAATGAACACCACAAGGAAATGTACCTCAAGCAAATTGTCTTT GGTGCAGTCTTGGTCGAGAATGAACACCACAAGGAAATGTACCTCAAGCAAATTGTCTTT CsLOX4-i so1/M13 GGTGCAGTCTTGGTCGAGAATGAACACCACAAGGAAATGTACCTCAAGCAAATTGTCTTT CsLOX4-i so3/M13 GGTGCAGTCTTGGTCGAGAATGAACACCACAAGGAAATGTACCTCAAGCAAATTGTCTTT

CsLOX4-iso 1 CsLOX4-iso2 CsLOX4-iso3 CATGGATTCCCTCCTGGTGGCCCTGTTCACGTCACTTGCAATTCATGGGTTGCTTCCAAA CATGGATTCCCTCCTGGTGGCCCTGTTCACGTCACTTGCAATTCATGGGTTGCTTCCAAA CATGGATTCCCTCCTGGTGGCCCTGTTCACGTCACTTGCAATTCATGGGTTGCTTCCAAA CsLOX4-i so 1/M13 CATGGATTCCCTCCTGGTGGCCCTGTTCACGTCACTTGCAATTCATGGGTTGCTTCCAAA CsLOX4-i so3/M13 CATGGATTCCCTCCTGGTGGCCCTGTTCACGTCACTTGCAATTCATGGGTTGCTTCCAAA

CsLOX4-iso 1 CsLOX4-iso2 CsLOX4-iso3 TTTCATAATCCTCACAAGAGAATTTTTTTCACCAATAAGTCATATTTGCCATCTCAAACA TTTCATAATCCTCACAAGAGAATTTTTTTCACCAATAAGTCATATTTGCCATCTCAAACA TTTCATAATCCTCACAAGAGAATTTTTTTCACCAATAAGG-

CsLOX4-i so1/M13 TTTCATAATCCTCACAAGAGAATTTTTTTCACCAATAAGTCATATTTGCCATCTCAAACA CsLOX4-i so3/M13 TTTCATAATCCTCACAAGAGAATTTTTTTCACCAATAAGG------------------

CsLOX4-iso 1

CsLOX4-i so2

CsLOX4-iso3

CsLOX4-i so 1/M13 CCAGATGGGTTGAAGAGGCTACGAGAGAAAGAGTTAGAAAATCTAAGGGGAAATGGGCAA CsLOX4-iso3/M13

CsLOX4-iso 1

CSLOX4-iso2

GGAAAGAGGAAAACACATGAAAGAATATACGATTATGATGTGTATAATGATATTGGTGAT CsLOX4-iso3 GGAAAGAGGAAAACACATGAAAGAATATACGATTATGATGTGTATAATGATATTGGTGAT CsLOX4-i so1/M13 GGAAAGAGGAAAATACATGAAAGAATATACGATTATGATGTGTATAATGATATTGGTGAT CsL0X4-i so3/M13 
CsLOX4-iso 1

CsLOX4-iso2

CsLOX4-i so3

CsLOX4-iso2/M13

CsLOX4-i so3/M13

CsLOX4-iso1

CsLOX4-i so2

CsLOX4-iso3

CsLOX4-i so1/M13 CGACGTTGCAGAACAGGTCGACCACGCTCTAAGACAGATCCAATGTCGGA-TCATGGTGC

CsLOX4-i so $3 /$ M13

CsLOX4-iso 1

CsLOX4-iso2

CsLOX4-i so3

CsLOX4-i so 1/M13 AGTGGTAGCATATACGTGCCAAGGGATGAGGCGTTCTCA-ATGTGAAGCAGCTAACATTT

CsL0X4-i so3/M13

CsL0X4-iso1

CsLOX4-iso2

CsLOX4-i so3

CsLOX4-iso1/M13 TCAGG

CsL0X4-iso3/M13

CsLOX4-iso 1

CsLOX4-iso2

CsLOX4-i so3

CsLOX4-i so $1 /$ M13

CsL0X4-i so2/M13

CsLOX4-i so3/M13

CsL0X4-i so1

CsLOX4-i so2

CsLOX4-iso3

CsL0X4-iso1/M13 G

CsLOX4-i so2/M13 GTTAACCTTCTTCCTCTTTCCAAAAATGGACTCTTGAAGGACCTCCTTCCCAGGCTTGTC CsL0X4-i so3/M13

CsLOX4-iso 1

CsLOX4-iso2

CsLOX4-iso3

CsLOX4-i so2/M13 AAGTTTGTCTCTGATGCTGAAGAAGGTCTTTTGCGCTTTGAGACCCCTGCAATGTTTGAA CsLOX4-i so3/M13

CsLOX4-iso 1

CsLOX4-i so2

AGAGACAAATTTTCTTGGCTTAGGGATGAAGAGTTCTCGAGGCAGACTCTTGGTGGTCTC AGAGACAAATTTTCTTGGCTTAGGGATGAAGAGTTCTCGAGGCAGACTCTTGGTGGTCTC

CsLOX4-iso3

CsLOX4-i so2/M13 AGAGACAAATTTTCTTGGCTTAGGGATGAAGAGTTCTCGAGGCAGACTCTTGGTGGTCTC CsLOX4-iso3/M13

CsLOX4-iso 1

CsLOX4-i so2

CsLOX4-i so3

CsLOX4-iso2/M13

CsLOX4-i so3/M13

CsL0X4-iso1

CsLOX4-i so2

CsLOX4-iso3

AAGTTTGTCTCTGATGCTGAAGAAGGTCTTTTGCGCTTTGAGACCCCTGCAATGTTTGAA AAGTTTGTCTCTGATGCTGAAGAAGGTCTTTTGCGCTTTGAGACCCCTGCAATGTTTGAA

CsLOX4-iso $2 / \mathrm{M} 13$

CsLOX4-i so3/M13

CsLOX4-iso 1

CsLOX4-iso2 $\begin{array}{ll}\text { CsLOX4-i so } 1 & \text { ATATACGGCCCACCAGAATCGGCAATCACCAAAGAGCTGATTGAGCGACAGATCCGGGGG } \\ \text { CsLOX4-i so2 } & \text { ATATACGGCCCACCAGAATCGGCAATCACCAAAGAGCTGATTGAGCGACAGATCAGGGGG } \\ \text { CsLOX4-i so2/M13 } & \text { ATATACGGCCGCCAGAATCGGCAATCACCAAAGAGCTGATTGAGCGACAGATCAGGGGG } \\ & \\ \text { CsLOX4-i so } 1 & \text { TTCATGACCCTTGAAGAGGCCTTGCAAACAAAGAAGCTTTTT-ATGCTGGATTACCATGA }\end{array}$

CsLOX4-iso1

CsLOX4-iso2 ATATACGGCCCACCAGAATCGGCAATCACCAAAGAGCTGATTGAGCGACAGATCCGGGGG
ATATACGGCCCACCAGAATCGGCAATCACCAAAGAGCTGATTGAGCGACAGATCAGGGGG
ATATACGGCCCGCCAGAATCGGCAATCACCAAAGAGCTGATTGAGCGACAGATCAGGGGG
TTCATGACCCTTGAAGAGGCCTTGCAAACAAAGAAGCTTTTT-ATGCTGGATTACCATGA ATATACGGCCCACCAGAATCGGCAATCACCAAAGAGCTGATTGAGCGACAGATCCGGGGG
ATATACGGCCCACCAGAATCGGCAATCACCAAAGAGCTGATTGAGCGACAGATCAGGGGG
ATATACGGCCCGCCAGAATCGGCAATCACCAAAGAGCTGATTGAGCGACAGATCAGGGGG
TTCATGACCCTTGAAGAGGCCTTGCAAACAAAGAAGCTTTTT-ATGCTGGATTACCATGA

AACCCATGCAGCATACAATTGGTCAAGGAATGGCCATTGAAGAGTAAACTTGACCTAAAG AACCCATGCAGCATACAATTGGTCAAGGAATGGCCATTGAAGAGTAAACTTGACCCAAAG

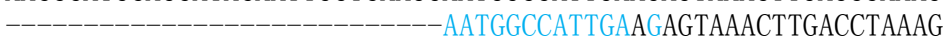
AACCCATGCAGCATACAATTGGTCAAGGAATGGCCATTGAAGAGTAAACTTGACCCGAAG

ATATACGGCCCACCAGAATCGGCAATCACCAAAGAGCTGATTGAGCGACAGATCCGGGG ATATACGGCCCACCAGAATCGGCAATCACCAAAGAGCTGATTGAGCGACAGATCAGGGGG ATATACGGCCCACCAGAATCGGCAATCACCAAAGAGCTGATTGAGCGACAGATCCGGGGG AACCCATGCAGCATACAATTGGTCAAGGAATGGCCATTGAAGAGTAAACTTGACCCGAAG TTCATGACCCTTGAAGAGGCCTTGCAAACAAAGAAGCTTTTTTATGCTGGATTACCATGA 
CsLOX4-i so2/M13 TTCATGACACTTGAAGAGGCCTTGCAAACAAAGAAGCTTTTT-ATGCTGGATTACCATGA

CsLOX4-iso1 CTTGTTGCTACCTTATGTGAACAAAGTAAGAGAGAGCAAAGGGACAGCACTGTATGGGTC CsLOX4-iso2 CTTGTTGCTACCTTATGTGAACAAAGTAAGAGAGAGCAAAGGGACAACACTGTATGGGTC CsLOX4-iso2/M13 CTTGTTGCTACCTTATGTGAACAAAGTAAGAGAGAGCAAAGGGACAACACTGTATGGGTC

CsLOX4-i so1 GAGGACGCTCTTCTTCTTAACACCTGATGGCACCTTGAGGCCTTTAGCCATAGAGCTGAC CsLOX4-i so2 GAGGACACTCTTCTTCTTAACACCTGATGGCACCTTGAGGCCTTTAGCCATAGAGCTGAC CsLOX4-i so2/M13 GAGGACGCTCTTCTTCTTAACACCTGATGACACCTTGAGGCCTTTAGCCATAGAGCTGAC

CsLOX4-iso1 TCGGCCACCAGTAGACGACAAGCCTCAGTGGAAGCAAGTGTTCACACCTACCGGGGATGC CsLOX4-i so2 TCGGCCACCAGTAGACGACAAGCCTCAGTGGAAGCAAGTGTTCACACCTACCGGGGATGC CsLOX4-i so2/M13 TCGGCCACCAGTAGACGACAAGCCTCAGTGGAAGCAAGTGTTCACACCTACCGGGGATGC

CsLOX4-i so 1 TACTGGCTGCTGGCTTTGGAGGCTTGCCAAGGTTCATGCCCTTGCTCATGACTCTGGTTA CsLOX4-i so2 TACTGGCTGCTGGCTTTGGAGGCTTGCCAAGGTTCATGCCCTTGCTCATGACTCTGGTTA CsLOX4-i so2/M13 TACTGGCTGCTGGCTTTGGAGGCTTGCCAAGGTTCATGCCCTTGCTCATGACTCTGGTTA

CsLOX4-iso 1 TCACCAGTTAGTCAGTCACTGGCTAAGAACTCACTGCGTGACAGAGCCTTACATAATTGC CsLOX4-i so2 TCACCAGTTAGTCAGTCACTGGCTAAGAACTCACTGCGTGACAGAGCCTTACATAATTGC CsLOX4-i so2/M13 TCACCAGTTAGTCAGTCACTGGCTAAGAACTCACTGCGTGACAGAGCCTTACATAATTGC

CsLOX4-i so1 GAGCAACAGGCAACTTAGTGCAATGCACCCGATTTACAAACTATTGCACCCTCATTTTCG CsLOX4-i so2 GAGCAACAGGCAACTTAGTGCAATGCACCCGATTTACAAACTATTGCACCCTCATTTTCG CsLOX4-i so2/M13 GAGCAACAGGCAACTTAGTGCAATGCACCCGATTTACAAACTATTGCACCCTCATTTTCG

CsLOX4-iso1 GTACACAATGGAGATCAATGCTTTGGCTCGACAAGCCCTTATTAATGCTGGTGGAATTAT CsLOX4-i so2 GTACACAATGGAGATCAATGCTTTGGCTCGACAAGCCCTTATTAATGCTGGTGGAATTAT CsLOX4-i so2/M13 GTACACAATGGAGATCAATGCTTTGGCTCGACAAGCCCTTATTAATGCTGGTGGAATTAT

CsLOX4-iso1 TGAGACTTGTTTCTCACCCAAAAAGTACTCTATCGAGCTTAGCTCTGTTGCCTATGATCA CsLOX4-i so2 TGAGACTTGTTTCTCACCCAAAAAGTACTCTATCGAGCTTAGCTCTGTTGCCTATGATCA CsLOX4-i so2/M13 TGAGACTTGTTTCTCACCCAAAAAGTACTCTATCGAGCTTAGCTCTGTTGCCTATGATCA

CsLOX4-iso1 ACAGTGGCGTTTCGATCTCCAAGCACTACCGGCTGACTTAATTAGCAGGGGAATGGCTAT CsLOX4-i so2 ACAGTGGCGTTTCGATCTCCAAGCACTACCGGCTGACTTAATTAGCAGGGGAATGGCTAT CsLOX4-i so2/M13 ACAGTGGCGTTTCGATCTCCAAGCACTACCGGCTGACTTAATTAGCAGGGGAATGGCTAT

CsLOX4-iso1 GGAGGATCCAACAGCTCTGCATGGCCTAAGGCTAACCATCGAGGACTACCCTTACGCGAG CsLOX4-i so2 GGAGGATCCAACAGCTCTGCATGGCCTAAGGCTAACCATCGAGGACTACCCTTACGCGAG CsLOX4-i so2/M13 GGAGGATCCAACAGCTCTGCATGGCCTAAGGCTAACCATCGAGGACTACCCTTACGCGAG

CsLOX4-iso1 CGATGGTCTGCTAGTTTGGGATGCCATAAAGCAATGGGTTACAGACTATGTCAAACACTA CsLOX4-i so2 CGATGGTCTGCTAGTTTGG-ATGCCATAAAGCAATGGGTTACAGACTATGTCAAACACTA CsLOX4-i so2/M13 CGATGGTCTGCTAGTTTGGGATGCCATAAAGCAATGGGTTACAGACTATGTCAAACACTA

CsLOX4-iso1 TTACCAAGATGCAAGCTTTGTACAGTCTGACAAAGAGCTTCAAGCATGGTGGACAGAAAT CsLOX4-iso2 TTACCAAGATGCAAGCTTTGTACAGTCTGACAAAGAGCTTCAAGCATGGTGGACAGAAAT CsL0X4-i so2/M13 TTACCAAGATGCAAGCTTTGTACAGTCTGACAAAGAGCTTCAAGCATGGTGGACAGAAAT

CsLOX4-iso1 CCGAACGGTAGGTCATGGTGACAAGAAAGACGAAACATGGTGGCCAGTGTTGAAAACCCC CsLOX4-iso2 CCGAACGGTAGGTCATGGTGACAAGAAAGACGAAACATGGTGGCCAGTGTTGAAAACCCC CsLOX4-i so2/M13 CCGAACGGTAGG

\section{CsADH8}

CSADH8-i so 1 AAAACCAAGGCCTGCGGGGTTTGCCACTCTGATCTTCACGTAATCAAAGGTGAACTTCCA CsADH8-i so2 AAAACCAAGGCCTGCGGGGTTTGCCACTCTGATCTTCACGTAATCAAAG----_--_--CsADH8-i so $1 /$ M13

CsADH8-iso2/M13 ATCAAAG

CsADH8-i so 1 TTTGCTAGCCCTTGTGTTGTGGGTCATGAGATCACAGGTGAGGTGGTTGAGCATGGGCCC CsADH8-i so2

CsADH8-i so $1 /$ M13

CsADH8-i so2/M13

CsADH8-iso 1

CTCACGGACACCAAAATCATTGAAAGATTTCCAGTCGGAGCTCATGTGATTGGAGCTTTC CsADH8-iso2 -ATTTCCAGTCGGAGCTCATGTGATTGGAGCTTTC

CsADH8-i so 1/M13 CTCACGGACACCAAAATCATTGAAAGATTTCCAGTCGGAGCTCATGTGATTGGAGCTTTC CsADH8-iso2/M13 -ATTTCCAGTCGGAGCTCATGTGATTGGAGCTTTC 
CsADH8-iso1 ATAATGCCTTGCGGTAGCTGTTTCTTCTGTAATAAGGGTCAAGATGACTTGTGTGAGGAT CsADH8-i so2 ATAATGC-TTGCGGTAGCTGTTTCTTCTGTAATAAGGGTCAAGATGACTTGTGTGAGGAT CsADH8-iso1/M13 ATAATGCCTTGCGGTAGCTGTTTCTTCTGTAATAAGGGTCAAGATGACCTGTGTGAGGAT CsADH8-iso2/M13 ATAATGCCTTGCGGTAGCTGTTTCTTCTGTAATAAGGGTCAAGATGACTTGTGTGAGGAT

CsADH8-i so 1 TTCTTCGCTTATAACCGCGCAAAAGGAACCCTTTATGACGGAGAAACCCGCTTGTTCCTC CsADH8-i so2 TTCTTCGCTTATAACCGCGCAAAAGGAACCCTTTATGACGGAGAAACCCGCTTGTTCCTC CsADH8-iso1/M13 TTCTTCGCTTATAACCGCGCAAAAGGAACCCTTTATGACGGAGAAACGCGCTTGTTCCTC CsADH8-iso2/M13 TTCTTCGCTTATAACCGCGCAAAAGGAACCCTTTATGACGGAGAAACGCGCTTGTTCCTC

CsADH8-iso1 CGCAACAGTGGCAAACCTGTATATATGTATAGCATGGGTGGCCTTGCTGATTTTTGTGTC CsADH8-i so2 CGCAACAGTGGCAAACCTGTATATATGTATAGCATGGGTGGCCTTGCTGATTTTTGTGTC CsADH8-i so 1/M13 CGCAACAGTGGCAAACCTGTATATATGTATAGCATGGGTGGCCTTGCTGATTTTTGTGTC CsADH8-i so2/M13 CGCAACAGTGGCAAACCTGTATATATGTATAGCATGGGTGGCCTTGCTGATTTTTGTGTC

CsADH8-iso1 GTGCCAGCACATGGTTTGTGCATTCTACCCGACACATTGCCGTATACAGAATCTGCTATT CsADH8-iso2 GTGCCAGCACATGGTTTGTGCATTCTACCCGACACATTGCCGTATACAGAATCTGCTATT CSADH8-i so 1/M13 GTGCCAGCACATGGTTTGTGCATTCTACCCGACACATTGCCGTATACAGAATCTGCTATT CsADH8-i so2/M13 GTGCCAGCACATGGTTTGTGCATTCTACCTGACACATTGCCGTATACAGAATCTGCTATT

CsADH8-i so 1 TTGGGATGTGCAGTTTTTACCGCATATGGTGCTATGGCTCATGCAGCTGAGGTTCGTCCT CsADH8-iso2 TTGGGATGTGCAGTTTTTACCGCATATGGTGCTATGGCTCATGCAGCTGAGGTTCATCCT CsADH8-i so 1/M13 TTGGGATGTGCAGTTTTTACTGCATATGGTGCCATGGCTCATGCAGCTGAGGTTCGTCCT CsADH8-i so2/M13 TTGGGATGTGCAGTTTTTACCGCATATGGTTCTATGGCTCATGCAGCTGAGGTTCATCCT

CsADH8-i so1 GGGGATACCGTTGCTGTGATTGGCATTGGGGGTGTTGGTTCTAGTTGTTTGCAGATAGCA CsADH8-iso2 GGG-ATACCATTGCTGTGATTGGCATTGGGGGTGTTGGTTCTAGTTGTTTGCAGATAGCA CsADH8-i so1/M13 GGGGATACCGTTGCTGTGATTGGCATTGGGGGTGTTGGTTCTAGTTGTTTGCAGATAGCA CsADH8-i so2/M13 GGGGATACCGTTGCTGTGATTGGCATTGGGGGTGTTGGTTCTAGTTGTTTGCAGATAGCA

CsADH8-i so1 CGGGCATTTGGCGCCTCTGAGATCATAGCTGTTGATGTACAAGACGATAAACTGCAGAAA CsADH8-i so2 CGGGCATTTGGCGCCTCTGAGATCATAGCTGTTGATGTACAAGACGATAAACTGCAGAAA CsADH8-i so 1/M13 CGGGCATTTGGCGCCTCTGAGATCATAGCTGTTGATGTACAAGACGATAAACTGCAGAAA CsADH8-i so2/M13 CGGGCATTTGGCGCCTCTGAGATCATAGCTGTTGATGTACAAGACGATAAACTGCAGAAA

CsADH8-iso1 GCAAAAATGCTTGGAGCTACACACACTGTAAATGCACAAAATGAGGATGCTGTTGAAAAG CsADH8-i so2 GCAAAAATGCTTGGAGCTACACACACTGTAAATGCACAAAATGAGGATGCTGTTGAAAAG CsADH8-i so 1/M13 GCAAAAATGCTTGGGGCTACACACACTGTAAATGCACAAAATGAGGATGCTGTTGAAAAG CsADH8-i so2/M13 GCAAAAATGCTTGGAGCTACACACACTGTAAATGCACAAAATGAGGATGCTGTTGAAAAG

CsADH8-iso1 ATTAGAGAAATAACTGGAGGAATGGGGGTAGACATTGCTGTGGAAGCCCTTGGAAAACCG CsADH8-i so2 ATTAGAGAAATAACTGGAGGAA-GGGGGTAGACATTGCTGTGGAAGCCCTTGGAAAACCG CsADH8-iso1/M13 ATTAGAGCAATAACTGGAGGAATGGGGGTAGACATTGCTGTGGAAGCCCTTGGAAAACCG CsADH8-iso2/M13 ATTAGAGAAATAACTGGAGGAATGGGGGTAGACATTGCTGTGGAAGCCCTTGGAAAACCG

CsADH8-iso1 CAGACATTTTTGCAGTGCACACAAAGTGTACGAGATGGAGGAAAAGCTGTAATGATTGGG CsADH8-iso2 CAGACATTTTTGCAGTGCACACAAAGTGTACGAGATGGAGGAAAAGCTGTAATGATTGGG CsADH8-i so1/M13 CAGACATTTTTGCAGTGCACACAAAGTGTACGAGATGGAGGAAAAGCTGTAATGATTGGG CsADH8-i so2/M13 CAGACATTTTTGCAGTGCACACAAAGTGTACGAGATGGAGGAAAAGCTGTAATGATTGGG

CsADH8-iso1 CTTACGCACTCTGCTGCCAGAGGGGAGATAGATATAAACCGTCTGGTTCGTAGGCAGATT CsADH8-i so2 CTTACGCACTCTGCTGCCAGAGGGGAGATAGATATAAACCGTCTGGTTCGTAGGCAGATT CsADH8-iso 1/M13 CTTACGCACTCTGCTGCCAGAGGGGAGATAGATATAAACCGTCTGGTTCGTAGGCAGATT CsADH8-iso2/M13 CTTACGCACTCTGCTG-

CsADH8-i so1 AAAATAATTGGCTCATACGGAGGCAGAGCAAGGCAGGATCTTCCAAAGTTGGTTAAGCTT CsADH8-i so2 AAAATAATTGGCTCATACGGAGGCAGAGCAAGGCAGGATCTTCCAAAGTTGGTTAAGCTT CsADH8-i so1/M13 AAAATAATTGGCTCATATGGAGGCAGAGCAAGGCAGGATCTTCCAAAGTTGGTTAAGCTT

CsADH8-iso 1 TCCGAAAGCGGTATCTTCAATCTCAGTGCTGCTGTTTCAAGAAAATGCAAAATCGAGGAT CSADH8-iso2 TCCGAAAGCGGTATCTTCAATCTCAGTGCTGCTGTTTCAAGAAAATGCAAAATCGAGGAT CsADH8-i so1/M13 TCCGAAAGCGGTATCTTCAACCTCAGTGCTGCTGTTTCAAGAAAATGCAAAATCGAGGAT

CsADH8-i so1 GCAAATGAAGTGTACCAA-GATCTCAACCGGGGCAGTATTGTTGGCGTGCTGTTGTTGAG CsADH8-i so2 GCAAATGAAGTGTACCAA-GATCTCAACCGGGGCAGTATTGTTGGCGTGCTGTTGTTGAG CsADH8-i so1/M13 GCAAATGAAGTGTACCAATGATCTCAACCGGGGCAGTATTGTTGGCGTGCTGTT------

Figure S3. Alignment of splice-variant transcripts with the sequences of the verified isoforms. 
Colored sequences indicate cloning primer.

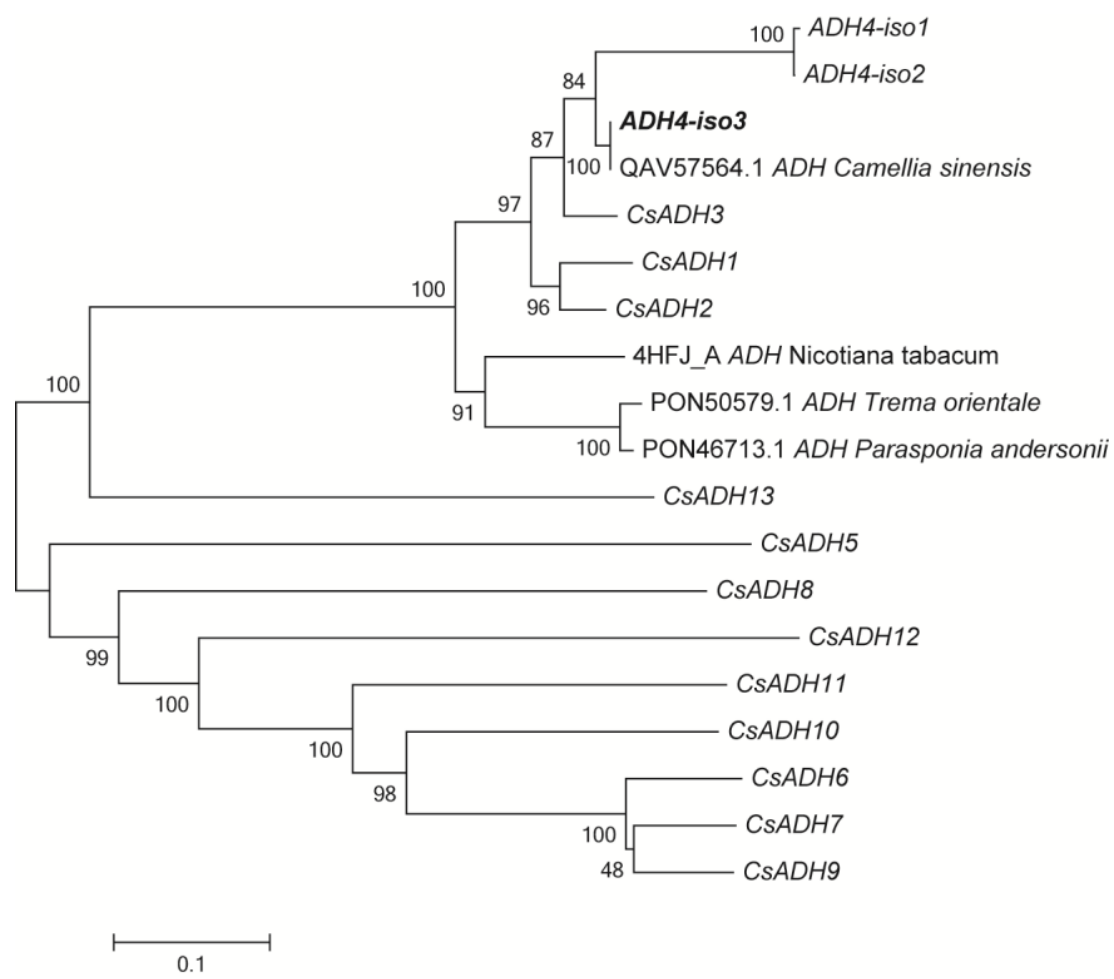

Figure S4. Phylogenetic analysis of CsADHs with several functionally characterized ADH genes from other plant species.

ATGGCGGTGGTGGGAGAGGAAGTGAGCAACAAACAGATCATTCTGAAGGAGTACGT GAGTGGTTTCCCCAAAGAGTCGGACATGGTGGTGAAGACTGGTACCATTCGTCTCCA GCTTCCAGAGGACTCGAAGAACGCCATTCTGGTCAAGAATCTCTACTTGTCTTGCGAT CCTTACATGCGTGGTCGCATGAGGAAGATGGAAGATAGCTACGTTGAGTCCTTCACTC CTGGTTCTCCTATAACTGGATATGGAGTGGCTAGAGTTTTGGATTCTGGACATCCAAA CTTTAAGAAAGGTGACTTGGTTTGGGGAATAACTGGATGGGAAGAGTATAGTGTCAT TAAAGCACAAGAGAGCGTATTTAAAATTCAAAATACAAATGTGGCTCTTTCTTATTAT ACAGGAATCCTTGGCATGCCTGGTATGACGGCCTATGTTGGTTTTTATGAGCTGTGCT CACCTAAGCAAGGAGAGTATGTTTTTGTTTCAGCTGCCTCTGGAGCTGTTGGTCAGCT TGTTGGGCAGTTTGCAAAGTTATTAGGCTGTTATGTTGTTGGAAGTGCCGGAACCAAA GAAAAGGTTGATTTGTTGAAGAACAAATTTGGGTTTGATGAAGCTTTTAACTATAAAG AGGAGGACTTGGAAGCAACTCTGAAAAGGTACTTTCCAAATGGCATTGATATTTACTT TGAAAATGTTGGAGGAAAGATGCTTGATGCAGTACTTCTCAACATGAGACTCCGCGG TCGCATTGCTATGTGTGGTATGATCTCACAGTACAACCTTGAACAACCCGAAGGTATT CACAACTTGTTTTGTCTTATCACAAAACGAGTCCGTATGGAAGGATTTGTAGTTCCCG ATTACTATCACATGTATCCAAAGTTTCTAGACATGATCTTGCCCCACATAAAAGAAGG AAAAATCACATACGTGGAAGACATAGCTGAAGGCCTAGAGAGTGCCCCAGCAGCTCT TATTGGGCTTTTTGTCGGTCACAATGTCGGCAAACAGGTAGTGGTTGTTGCTCGTGAA TGA

Figure S5. Sequences of CsADH4-iso3 open reading frames. 